\title{
Near-Optimal Low-Complexity Sequence Detection for Clipped DCO-OFDM
}

\author{
Jiandong Tan, Zhaocheng Wang, Senior Member, IEEE, Qi Wang, and Linglong Dai, Senior Member, IEEE
}

\begin{abstract}
The inherent high peak-to-average power ratio issue of dc-biased optical orthogonal frequency division multiplexing (DCO-OFDM) is sensitive to the limited dynamic region of light emitting diode component and prone to clipping distortion, which deteriorates the performance of visible light communication systems. This letter proposes a maximum likelihood sequence detection (MLSD) method for the clipped DCO-OFDM, whereas the double-sided clipping characteristic is incorporated to improve the performance. Besides that, a near-optimal low-complexity MLSD method is presented to reduce the calculation complexity. Simulations demonstrate that the proposed low-complexity MLSD receiver could approach the performance of ideal case of non-clipped DCO-OFDM.
\end{abstract}

Index Terms-Visible light communication (VLC), dc-biased orthogonal frequency-division multiplexing (DCO-OFDM), maximum likelihood sequence detection (MLSD), clipping distortion.

\section{INTRODUCTION}

$\mathbf{S}$ INCE LIGHT emitting diodes (LED) are massively adopted in commercial illumination market, LED-based visible light communication (VLC) is becoming a promising technique to complement the conventional radio frequency (RF) systems since VLC exhibits some advantages of high rate downlink transmission and broad unlicensed spectrum with unaffected illumination functionality [1]. To enhance the spectral efficiency and resist the inter-symbol interference (ISI), orthogonal frequency-division multiplexing (OFDM) scheme is recommended for VLC multiplesubcarrier system [2]. Since the intensity modulation with direct detection (IM/DD) [3] is deployed in typical VLC systems, optical OFDM signals are modulated on the intensity of the emitted light and restricted to be real-valued and non-negative.

DC-biased OFDM (DCO-OFDM) [4] is one of the popular modulation schemes for VLC systems. In DCO-OFDM,

Manuscript received July 7, 2015; revised October 3, 2015; accepted October 16, 2015. Date of publication October 26, 2015; date of current version December 21, 2015. This work was supported in part by the National Key Basic Research Program of China under Grant 2013CB329203, in part by the National Natural Science Foundation of China under Grant 61271266, in part by the Shenzhen Visible Light Communication System Key Laboratory under Grant ZDSYS20140512114229398, in part by the Shenzhen Wireless over Visible Light Communication Technology Engineering Laboratory under Grant SDRC[2012]No.1440, and in part by the Shenzhen Peacock Plan under Grant 1108170036003286.

The authors are with the Tsinghua National Laboratory for Information Science and Technology, Department of Electronic Engineering, Tsinghua University, Beijing 100084, China (e-mail: tanjd09@gmail.com; zcwang@ tsinghua.edu.cn; qiwang11@mails.tsinghua.edu.cn; daill@tsinghua.edu.cn).

Color versions of one or more of the figures in this letter are available online at http://ieeexplore.ieee.org.

Digital Object Identifier 10.1109/LPT.2015.2493169 the Hermitian symmetry is introduced in frequency domain to satisfy the real-valued constraint and DC bias is added to convert the time-domain signals to be non-negative. In terms of the nonlinear transfer characteristics of LED, the time-domain DCO-OFDM amplitudes exceeding the LED dynamic region are clipped, which leads to nonlinear distortion inevitably. At the conventional receiver for DCO-OFDM, the clipping distortion is modeled as Gaussian non-correlated noise [5], [6] and the received symbol on each subcarrier is detected separately by maximum likelihood detection (MLD) method. After fast Fourier transformation (FFT) operation, the clipping distortion converted into the frequency domain is correlated with the symbol sequence on all subcarriers. The conventional MLD method for each subcarrier neglects this phenomenon and leads to a reduced equivalent signal-to-noise ratio (SNR), which deteriorates the performance of VLC systems.

PAPR reduction technique is considered to mitigate the clipping distortion. In the literature of [7]-[10], several low PAPR algorithms designed for DCO-OFDM are proposed to reduce the peak amplitude and the power of clipping noise accordingly. These methods introduce the additional complexity for both the transmitter and receiver and the negative effects such as data rate loss and bit error rate (BER) performance degradation could not be neglected. Besides that, optimizing DC biasing and scaling factor is considered in order to minimize the effect of clipping distortion [11], [12]. However, only limited performance gain is achieved since the clipping noise is not completely removed. The literature [13] proposes a decision-aided reconstruction method to detect the clipped OFDM signals iteratively, which is based on the conventional MLD and requires extra IFFT/FFT operations per iteration at the receiver. This letter proposes a nearoptimal low-complexity detector using maximum likelihood sequence detection (MLSD) criterion to mitigate the LED clipping distortion, whereas it could be recovered according to the detected symbol sequence rather than simply regarded as Gaussian noise. Marginal performance loss compared with the ideal case of non-clipped DCO-OFDM is verified by simulation results.

\section{SySTEM MODEL}

At the transmitter, assuming $N_{\text {used }}$ subcarriers of $N$-point DCO-OFDM are occupied, the input bits are mapped into data symbol sequence $\mathbf{X}=\left[X_{1}, X_{2}, \ldots, X_{N_{\text {used }}}\right] \in \mathcal{X}^{N_{\text {used }}}$, where $\mathcal{X}$ denotes the constellation set. To guarantee the realvalued time-domain signals, the data symbols are assigned on the associated subcarriers in Hermitian symmetry to acquire 
the frequency-domain symbol vector $\mathbf{X}_{\mathrm{DCO}}$. The Hermitian symmetry operation $H S(\cdot)$ is defined as

$$
\begin{aligned}
\mathbf{X}_{\mathrm{DCO}} & =H S(\mathbf{X}) \\
& =\left[0, X_{1}, \ldots, X_{N_{\text {used }}}, 0, \ldots, 0, X_{N_{\text {used }}}^{*}, \ldots, X_{1}^{*}\right] .
\end{aligned}
$$

The time-domain signals $\mathbf{x}_{\mathrm{DCO}}=\operatorname{IFFT}\left(\mathbf{X}_{\mathrm{DCO}}\right)$ are calculated by $N$-point IFFT operation. Denoting $x_{\mathrm{DCO}, n}$ as the $n$-th element of $\mathbf{x}_{\mathrm{DCO}}$ and $X_{\mathrm{DCO}, k}$ as the $k$-th element of $\mathbf{X}_{\mathrm{DCO}}$, $x_{\mathrm{DCO}, n}$ could be expressed as

$x_{\mathrm{DCO}, n}=\frac{1}{\sqrt{N}} \sum_{k=0}^{N-1} X_{\mathrm{DCO}, k} \exp \left(j \frac{2 \pi k n}{N}\right), n=0,1, . ., N-1$.

$\mathbf{x}_{\text {DCO }}$ is biased with a constant DC voltage $B_{\text {DC }}$ and fed to drive the LED component. Since the LED transfer characteristic is non-linear, the pre-distorter is introduced, whereas the LED nonlinearity can be modeled as doublesided clipping [14]. Hence, the transmitted optical signals $\mathbf{x}$ are acquired by clipping the amplitudes of the DC-biased OFDM signals with LED dynamic region $[0, A]$ as

$$
\mathbf{x}=\left\lfloor\mathbf{x}_{\mathrm{DCO}}+B_{\mathrm{DC}}\right\rfloor_{A},
$$

where the clipping operation $\lfloor\cdot\rfloor_{A}$ is given by

$$
\lfloor z\rfloor_{A}= \begin{cases}0, & z<0 \\ A, & z>A \\ z, & \text { else. }\end{cases}
$$

The level of clipping distortion is determined by the distribution of $\mathbf{x}_{\mathrm{DCO}}$, the values of $A$ and $B_{\mathrm{DC}}$. Since $B_{\mathrm{DC}}$ is set as half of $A$ in order to take full advantage of LED dynamic region, the clipping level can be presented as [7]

$$
C L=10 \log _{10} \frac{A^{2}}{E\left\{x_{\mathrm{DCO}, n}^{2}\right\}} \mathrm{dB} .
$$

At the receiver, the avalanche photodiode (APD) captures the emitted optical signals and transfers them into electric signals r. The additive white Gaussian noise (AWGN) channel is assumed and we have [14]

$$
\mathbf{r}=\mathbf{x}+\mathbf{n},
$$

where $\mathbf{n}$ satisfies $N$-dimension independent and identical Gaussian distribution with zero mean and variance of $\sigma^{2}$. At the conventional receiver for DCO-OFDM, the clipping distortion is modeled as uncorrelated noise [6]. Hence, the receiver converts $\mathbf{r}$ into $\mathbf{R}$ directly after FFT operation and detects $\mathbf{R}$ separately on each occupied subcarrier. According to MLD criterion, the $k$-th data symbol $X_{k}^{r}$ is demodulated as

$$
X_{k}^{r}=\underset{\bar{X}_{k} \in \mathcal{X}}{\arg \min }\left\{\left\|R_{k}-\bar{X}_{k}\right\|^{2}\right\}, \quad k=1,2, \ldots, N_{\text {used }},
$$

where $R_{k}$ and $\bar{X}_{k}$ denotes the $k$-th element of $\mathbf{R}$ and the candidate symbol sequence $\overline{\mathbf{X}}$ respectively and $\|\cdot\|$ indicates the Euclidean distance. Compared to the ideal non-clipped DCO-OFDM, the equivalent SNR of the conventional MLD method is decreased due to the clipping noise, which leads to an inevitable performance loss [6].

\section{Proposed Low-Complexity Detection}

In this section, a MLSD detector is firstly proposed to mitigate the double-sided clipping distortion. After that, a near-optimal low-complexity detection is proposed to reduce the calculation complexity significantly with negligible performance loss.

\section{A. Optimal MLSD Criterion}

The transmitted symbols on the occupied subcarriers are separately detected at the conventional MLD receiver, whereas the clipping distortion is modeled as static Gaussian noise. Actually, the dynamic clipping distortion can be recovered according to the $N_{\text {used }}$-point sequence of the estimated candidate symbols $\overline{\mathbf{X}} \in \mathcal{X}^{N_{\text {used }}}$ by repeating the calculation of Eq. (1)-(3). Hence, the optimal MLSD receiver should detect the candidate sequence $\overline{\mathbf{X}}$ for each OFDM block in order to estimate the associated clipping distortion. Assuming that the LED dynamic region $[0, A]$ and DC bias voltage $B_{\mathrm{DC}}$ are known at the receiver, the likelihood of $\mathbf{r}$ on condition that the transmitted symbol sequence $\mathbf{X}$ equals to the estimated candidate $\overline{\mathbf{X}}$ is formulated according to the Gaussian distribution of $\mathbf{n}$ as

$$
P(\mathbf{r} \mid \mathbf{X}=\overline{\mathbf{X}})=\frac{1}{\left(2 \pi \sigma^{2}\right)^{\frac{N}{2}}} \exp \left(-\frac{1}{2 \sigma^{2}}\left\|\mathbf{r}-\left\lfloor\mathbf{x}^{r}(\overline{\mathbf{X}})\right\rfloor_{A}\right\|^{2}\right) \text {. }
$$

The regenerated time-domain DCO-OFDM signal $\mathbf{x}^{r}(\overline{\mathbf{X}})$ is acquired by

$$
\mathbf{x}^{r}(\overline{\mathbf{X}})=\operatorname{IFFT}(\operatorname{HS}(\overline{\mathbf{X}}))+B_{\mathrm{DC}} .
$$

The optimum symbol estimated sequence $\mathbf{X}_{M L S D}^{r}$ is computed through the maximization of the likelihood. According to (8), MLSD criterion is simply derived as

$$
\mathbf{X}_{M L S D}^{r}=\underset{\overline{\mathbf{X}} \in \mathcal{X}^{N_{\text {used }}}}{\arg \min }\left\{\left\|\mathbf{r}-\left\lfloor\mathbf{x}^{r}(\overline{\mathbf{X}})\right\rfloor_{A}\right\|^{2}\right\} .
$$

For readability, the square of Euclidean distance $d^{2}(\overline{\mathbf{X}})=$ $\left\|\mathbf{r}-\left\lfloor\mathbf{x}^{r}(\overline{\mathbf{X}})\right\rfloor_{A}\right\|^{2}$ indicates the MLSD metric of the candidate sequence $\overline{\mathbf{X}}$ in (10). With regard to the $M$-ary constellation set $\mathcal{X}$, the total number of traversing the arbitrary $\overline{\mathbf{X}}$ for minimization of $d^{2}(\overline{\mathbf{X}})$ is as high as $M^{N_{\text {used }}}$. Moreover, the optimal solution of (10) imposes $M^{N_{\text {used }}}$ IFFT operations to regenerate $\mathbf{x}^{r}(\overline{\mathbf{X}})$ and $M^{N_{\text {used }}}$ computations of the Euclidean distance. Due to the extremely high complexity of $O\left(M^{N_{\text {used }}}\right)$, the optimal MLSD method in (10) is not feasible for practical hardware implementation. Hence, a low-complexity detection using the MLSD criterion is proposed below, which could provide the near-optimal performance.

\section{B. Near-Optimal Low-Complexity Detection}

As is depicted in Fig. 1, the clipping distortions gather around the constellation when a low clipping level of $11 \mathrm{~dB}$ is adopted. By taking AWGN into account, the specific candidate symbol $\bar{X}_{k}$ acquired from the conventional MLD method in (7) falls on the neighborhood of the transmitted 


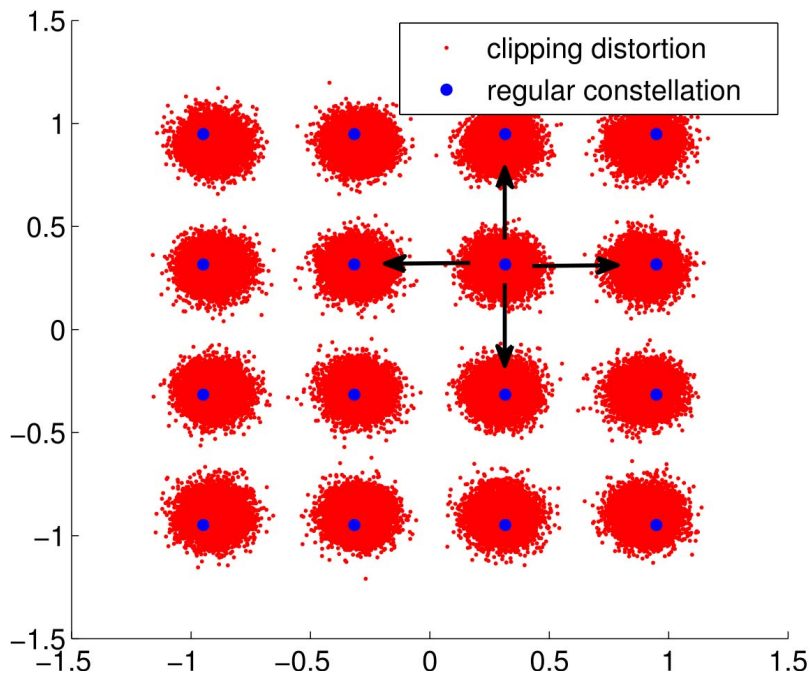

Fig. 1. The impact of clipping distortion to 16QAM constellation with $\mathrm{CL}=11 \mathrm{~dB}$ in DCO-OFDM.

constellation $X_{k}$ most probably rather than spreads to the whole constellation set.

Based on the distribution characteristic of clipping distortion, a low-complexity MLSD is proposed. The original candidate symbol sequence $\overline{\mathbf{X}}_{o}$ is acquired according to the conventional MLD method in (7) to update the estimated sequence by $\mathbf{X}^{r}$ with $\overline{\mathbf{X}}_{o}$. On the 1-st subcarrier, the first symbol $X_{1}^{r}$ is changed among its neighborhood constellations and other symbols in $\mathbf{X}^{r}$ remain unchanged. The associated time-domain DCO-OFDM signals are regenerated to compute the MLSD metric in (10). The symbol with the lowest MLSD metric is selected as the candidate $X_{1}^{r}$ for updating the sequence $\mathbf{X}^{r}$. The candidate symbol selection is repeated on the next subcarrier sequentially. After all the subcarriers are processed, the algorithm returns to detect the symbol on the 1-st subcarrier and the above step is repeated iteratively. The low-complexity detection algorithm is summarized as following:

1) Pre-process: For arbitrary constellation $X \in \mathcal{X}$, calculate its $m$ neighborhood constellations $\mathcal{X}(X, 0)$, $\mathcal{X}(X, 1), \ldots, \mathcal{X}(X, m-1)$ with the shortest Euclidean distance from $X$ and store all the subsets by lookup table. As is shown in Fig. 1, the error estimated symbol acquired from the conventional MLD falls on the 4 neighborhood constellations most probably. Hence, $m=4$ is utilized in this letter;

2) Initialization: the original candidate symbol sequence $\overline{\mathbf{X}}_{o}$ is simply detected with the conventional MLD criterion in (7) and the the estimated sequence is updated by $\mathbf{X}^{r}=\overline{\mathbf{X}}_{o}$. Reconstruct the time-domain DCO-OFDM signal $\mathbf{x}^{r}\left(\mathbf{X}^{r}\right)$ and calculate the MLSD metric $d^{2}\left(\mathbf{X}^{r}\right)$;

3) Set the iterator $i, l=0, k=1$;

4) Extract all the neighborhood constellations of $X_{k}^{r}$ from the look-up table and denote $X_{k, i}^{r}=\mathcal{X}\left(X_{k}^{r}, i\right)$;

5) Substitute the symbol $X_{k}^{r}$ with its $i$-th neighborhood constellation $X_{k, i}^{r}$ on the $k$-th subcarrier and fix other subcarriers in order to generate the candidate sequence $\overline{\mathbf{X}}$. Since there exists only one different subcarrier between $\mathbf{X}^{r}$ and $\overline{\mathbf{X}}$, the $n$-th element of the candidate time-domain
DCO-OFDM signal $x_{n}^{r}(\overline{\mathbf{X}})$ can be simply formulated as:

$$
\begin{aligned}
x_{n}^{r}(\overline{\mathbf{X}})= & x_{n}^{r}\left(\mathbf{X}^{r}\right) \\
& +\frac{2}{\sqrt{N}} \operatorname{Re}\left(\left(X_{k, i}^{r}-X_{k}^{r}\right) \exp j\left(\frac{2 \pi}{N} k n\right)\right), \\
n=0,1, \ldots N-1 . & (11)
\end{aligned}
$$

After $x_{n}^{r}(\overline{\mathbf{X}})$ is regenerated, calculate the MLSD metric of the candidate sequence $d^{2}(\overline{\mathbf{X}})$ according to (10). If $d^{2}(\overline{\mathbf{X}})<d^{2}\left(\mathbf{X}^{r}\right)$, update $\mathbf{X}^{r}, \mathbf{x}^{r}\left(\mathbf{X}^{r}\right)$ and $d^{2}\left(\mathbf{X}^{r}\right)$ with $\overline{\mathbf{X}}, \mathbf{x}^{r}(\overline{\mathbf{X}})$ and $d^{2}(\overline{\mathbf{X}})$ respectively;

6) Update the iterator $i=i+1$. If $i<m$, return to step 5); otherwise, reset $i=0$ and proceed to next step;

7) Update the iterator $k=k+1$. If $k \leqslant N_{\text {used }}$, return to step 4); otherwise, reset $k=1$ and proceed to next step;

8) Update the iterator $l=l+1$. If $l<L$, where $L$ denotes the maximum number of iterations, return to step 4); otherwise, terminate.

\section{Complexity Analysis}

As demonstrated in Section III-A, the optimal MLSD imposes $M^{N_{\text {used }}}$ IFFT operations and $M^{N_{\text {used }}}$ computations of the Euclidean distance to traverse all the possible candidate symbol sequence. Since the traversal complexity of $O\left(M^{N_{\text {used }}}\right)$ is extremely high, the optimal MLSD detector could not be directly applied in practical DCO-OFDM VLC systems especially when large subcarrier number is adopted.

For the low-complexity detection, in terms of the storage cost, the pre-process step consumes a look-up table to store $m M$ neighborhood constellations. The initialization step has the same complexity of estimating $\mathbf{X}^{r}$ as the conventional MLD method and only requires an additional IFFT operation and the computation of the Euclidean distance in calculation of MLSD metric. The steps 3)-8) include $m L N_{\text {used }}$ iterations of selecting the candidate symbol on the occupied subcarrier. In each iteration, the time-domain DCO-OFDM signal is regenerated according to equation (11), whereas no extra IFFT operation is required, and the MLSD metric is acquired by computing the Euclidean distance. In summary, the lowcomplexity detection consumes only 1 additional IFFT operation and $m L N_{\text {used }}+1$ computations of the Euclidean distance compared with the conventional MLD method and reduces the calculation complexity of solving the MLSD criterion from $O\left(M^{N_{\text {used }}}\right)$ to $O\left(m L N_{\text {used }}\right)$. Furthermore, the coefficient $m$ and $L$ can be set at the low level when the BER performance approaches the ideal case of non-clipped DCO-OFDM, which is illustrated in next section.

\section{Simulation Results}

In this section, simulation results are presented to evaluate the BER performance of the proposed low-complexity MLSD in DCO-OFDM VLC systems. The subcarrier number $N$ was set as 512 and $N_{\text {used }}=200$ subcarriers were occupied. At the transmitter, quadrature amplitude modulation (QAM) constellations with different orders are selected as the modulation schemes. The LED nonlinear characteristic was modeled as the doubled-side clipping in (4), where the DC bias voltage $B_{\mathrm{DC}}$ was set as $A / 2$ in order to balance the dynamic region [12]. As for 16QAM, 64QAM and 256QAM 


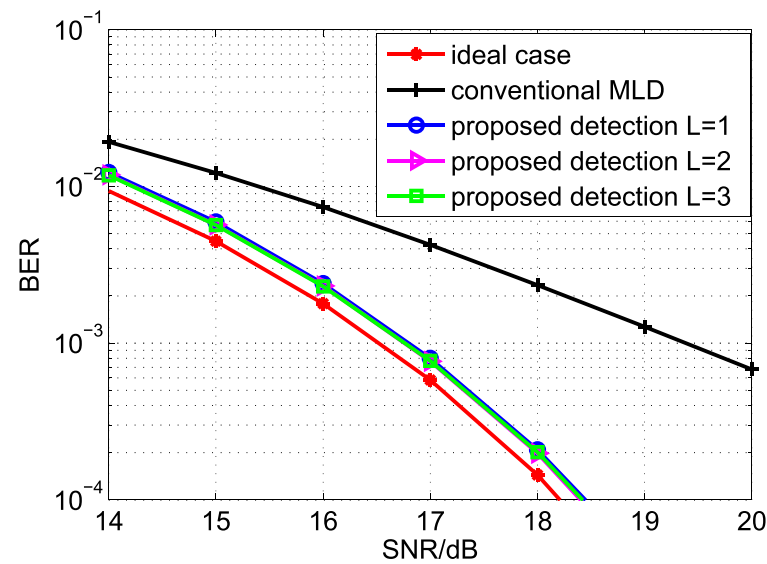

Fig. 2. BER performance of 16QAM for the conventional MLD, the proposed low-complexity detection and the ideal case (non-clipped DCO-OFDM).

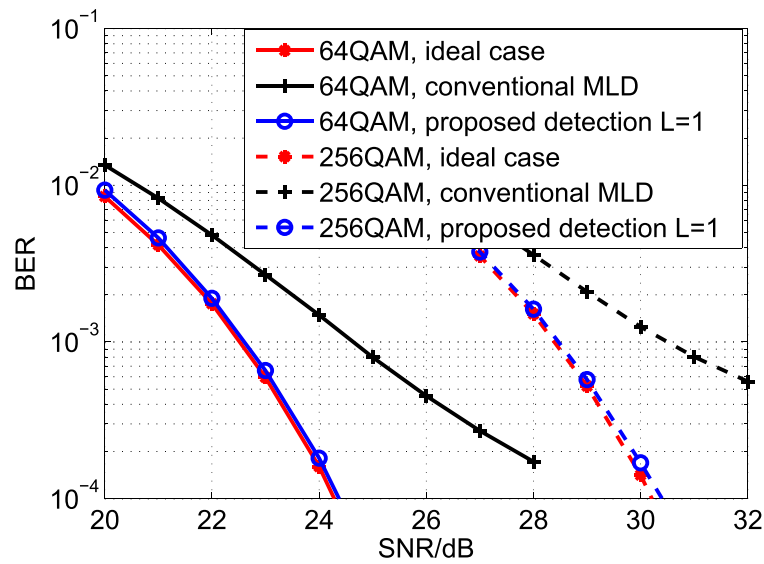

Fig. 3. BER performance of 64QAM and 256QAM for the conventional MLD, the proposed low-complexity detection and the ideal case (non-clipped DCO-OFDM).

constellation, the clipping levels were set as $11 \mathrm{~dB}, 13 \mathrm{~dB}$ and $14.4 \mathrm{~dB}$ respectively in order to handle the clipping distortion within a proper range. The minimum effective clipping levels of the proposed detection for the above constellations are recommended as $9 \mathrm{~dB}, 11 \mathrm{~dB}$, and $13 \mathrm{~dB}$, respectively. At the receiver, both the conventional MLD and the proposed lowcomplexity detection using MLSD criterion were evaluated. Besides that, BER curve of the ideal case without nonlinearity was also included as the benchmark, which represents the lower bound of the optimal MLSD method. Considering that the complexity should be reduced at the minimum level, the size of neighborhood constellation subset $m$ was set as 4 and the maximum iteration number $L$ was selected as 3 .

Fig. 2 demonstrates the BER performance comparison between the conventional MLD and the proposed low-complexity detection using MLSD criterion in 16QAM DCO-OFDM VLC systems. In this case, a low clipping level $C L=11 \mathrm{~dB}$ is adopted, which leads to a performance loss of $2.9 \mathrm{~dB}$ at $\mathrm{BER}=10^{-3}$ for the conventional MLD receiver. It indicates that the proposed low-complexity detection converges at the maximum iteration number $L=1$ and the gap with the ideal case is as low as $0.3 \mathrm{~dB}$. The high-order constellation schemes 64QAM and 256QAM are investigated in Fig. 3. For both modulation schemes, the conventional MLD method suffers a similar performance degradation of
$2.1 \mathrm{~dB}$ at BER $=10^{-3}$ with the $C L$ set as $13 \mathrm{~dB}$ and $14.4 \mathrm{~dB}$ respectively. In contrast, the proposed low-complexity detection with only $L=1$ iteration approaches BER performance of the ideal case and has a performance gain of $2.0 \mathrm{~dB}$ at $\mathrm{BER}=10^{-3}$ compared to the conventional MLD receiver. It is concluded that the proposed low-complexity detection achieves near-optimal performance and proves to be feasible for high order modulation schemes.

\section{CONCLUSION}

In this letter, we propose a near-optimal low-complexity MLSD scheme for DCO-OFDM VLC systems to mitigate the clipping distortion induced by LED nonlinearity. At the conventional MLD receiver, the data symbol is detected on each subcarrier separately, which is simple but leads to high performance loss. Hence, we propose a MLSD criterion to jointly detect the symbol sequence over all the subcarriers incorporating the LED nonlinear characteristic. In order to reduce the extremely high complexity of the optimal MLSD method, a low-complexity detection using MLSD criterion is also developed. Simulation results demonstrate that BER performance of the proposed near-optimal low-complexity MLSD method approaches the ideal case of non-clipped DCO-OFDM and reduces the negative effects of LED clipping distortion significantly.

\section{REFERENCES}

[1] A. Jovicic, J. Li, and T. Richardson, "Visible light communication: Opportunities, challenges and the path to market," IEEE Commun. Mag., vol. 51, no. 12, pp. 26-32, Dec. 2013.

[2] Q. Wang, Z. Wang, and L. Dai, "Asymmetrical hybrid optical OFDM for visible light communications with dimming control," IEEE Photon. Technol. Lett., vol. 27, no. 9, pp. 974-977, May 1, 2015.

[3] J. M. Kahn and J. R. Barry, "Wireless infrared communications," Proc. IEEE, vol. 85, no. 2, pp. 265-298, Feb. 1997.

[4] J. B. Carruthers and J. M. Kahn, "Multiple-subcarrier modulation for nondirected wireless infrared communication," IEEE J. Sel. Areas Commun., vol. 14, no. 3, pp. 538-546, Apr. 1996.

[5] Q. Wang, C. Qian, X. Guo, Z. Wang, D. G. Cunningham, and I. H. White, "Layered ACO-OFDM for intensity-modulated directdetection optical wireless transmission," Opt. Exp., vol. 23, no. 9, pp. 12382-12393, May 2015.

[6] S. Dimitrov, S. Sinanovic, and H. Haas, "Clipping noise in OFDMbased optical wireless communication systems," IEEE Trans. Commun., vol. 60, no. 4, pp. 1072-1081, Apr. 2012.

[7] Z. Yu, R. J. Baxley, and G. T. Zhou, "Iterative clipping for PAPR reduction in visible light OFDM communications," in Proc. IEEE MILCOM, Baltimore, MD, USA, Oct. 2014, pp. 1681-1686.

[8] Ö. Bulakçi, M. Schuster, C.-A. Bunge, and B. Spinnler, "Precoding based peak-to-average power ratio reduction for optical OFDM demonstrated on compatible single-sideband modulation with direct detection," in Proc. IEEE OFC/NFOEC, San Diego, CA, USA, Feb. 2008, pp. 1-3.

[9] W. O. Popoola, Z. Ghassemlooy, and B. G. Stewart, "Pilot-assisted PAPR reduction technique for optical OFDM communication systems," J. Lightw. Technol., vol. 32, no. 7, pp. 1374-1382, Apr. 1, 2014.

[10] H. Zhang, Y. Yuan, and W. Xu, "PAPR reduction for DCO-OFDM visible light communications via semidefinite relaxation," IEEE Photon. Technol. Lett., vol. 26, no. 17, pp. 1718-1721, Sep. 1, 2014.

[11] M. Zhang and Z. Zhang, "An optimum DC-biasing for DCO-OFDM system," IEEE Commun. Lett., vol. 18, no. 8, pp. 1351-1354, Aug. 2014

[12] Z. Wang, Q. Wang, S. Chen, and L. Hanzo, "An adaptive scaling and biasing scheme for OFDM-based visible light communication systems," Opt. Exp., vol. 22, no. 10, pp. 12707-12715, May 2014.

[13] D. Kim and G. L. Stübler, "Clipping noise mitigation for OFDM by decision-aided reconstruction," IEEE Commun. Lett., vol. 3, no. 1, pp. 4-6, Jan. 1999.

[14] S. Dimitrov and H. Haas, "Information rate of OFDM-based optical wireless communication systems with nonlinear distortion," J. Lightw. Technol., vol. 31, no. 6, pp. 918-929, Mar. 15, 2013. 